\title{
Combining epigenetic and clinicopathological variables improves specificity in prognostic prediction in clear cell renal cell carcinoma
}

Emma Andersson-Evelönn ${ }^{1 \dagger}$, Linda Vidman ${ }^{2 \dagger}$, David Källberg ${ }^{2,3}$, Mattias Landfors ${ }^{1}$, Xijia Liu ${ }^{2}$, Börje Ljungberg ${ }^{4}$, Magnus Hultdin ${ }^{1}$, Patrik Rydén ${ }^{2^{*+}}$ and Sofie Degerman ${ }^{1,5^{*}+(0)}$

\begin{abstract}
Background: Metastasized clear cell renal cell carcinoma (ccRCC) is associated with a poor prognosis. Almost onethird of patients with non-metastatic tumors at diagnosis will later progress with metastatic disease. These patients need to be identified already at diagnosis, to undertake closer follow up and/or adjuvant treatment. Today, clinicopathological variables are used to risk classify patients, but molecular biomarkers are needed to improve risk classification to identify the high-risk patients which will benefit most from modern adjuvant therapies. Interestingly, DNA methylation profiling has emerged as a promising prognostic biomarker in ccRCC. This study aimed to derive a model for prediction of tumor progression after nephrectomy in non-metastatic ccRCC by combining DNA methylation profiling with clinicopathological variables.
\end{abstract}

Methods: A novel cluster analysis approach (Directed Cluster Analysis) was used to identify molecular biomarkers from genome-wide methylation array data. These novel DNA methylation biomarkers, together with previously identified CpG-site biomarkers and clinicopathological variables, were used to derive predictive classifiers for tumor progression.

Results: The "triple classifier" which included both novel and previously identified DNA methylation biomarkers together with clinicopathological variables predicted tumor progression more accurately than the currently used Mayo scoring system, by increasing the specificity from $50 \%$ in Mayo to $64 \%$ in our triple classifier at $85 \%$ fixed sensitivity. The cumulative incidence of progress $\left({ }_{p} \mathrm{ClP}_{5 y \mathrm{r}}\right)$ was $7.5 \%$ in low-risk vs $44.7 \%$ in high-risk in $\mathrm{M0}$ patients classified by the triple classifier at diagnosis.

Conclusions: The triple classifier panel that combines clinicopathological variables with genome-wide methylation data has the potential to improve specificity in prognosis prediction for patients with non-metastatic ccRCC.

Keywords: Clear cell renal cell carcinoma, Classification, DNA methylation, Prognosis, Directed cluster analysis

\footnotetext{
*Correspondence: patrik.ryden@umu.se; sofie.degerman@umu.se

${ }^{\dagger}$ Emma Andersson-Evelönn and Linda Vidman contributed equally as first

authors, and Patrik Rydén and Sofie Degerman contributed equally as last authors

${ }^{1}$ Department of Medical Biosciences, Pathology, Umeå University, 901

87 Umeå, Sweden

${ }^{2}$ Department of Mathematics and Mathematical Statistics, Umeå

University, 90187 Umeå, Sweden

Full list of author information is available at the end of the article
}

\section{$\triangle B M C$}

The Author(s) 2020. This article is licensed under a Creative Commons Attribution 4.0 International License, which permits use, sharing, adaptation, distribution and reproduction in any medium or format, as long as you give appropriate credit to the original author(s) and the source, provide a link to the Creative Commons licence, and indicate if changes were made. The images or other third party material in this article are included in the article's Creative Commons licence, unless indicated otherwise in a credit line to the material. If material is not included in the article's Creative Commons licence and your intended use is not permitted by statutory regulation or exceeds the permitted use, you will need to obtain permission directly from the copyright holder. To view a copy of this licence, visit http://creativeco mmons.org/licenses/by/4.0/. The Creative Commons Public Domain Dedication waiver (http://creativecommons.org/publicdomain/ zero/1.0/) applies to the data made available in this article, unless otherwise stated in a credit line to the data. 


\section{Background}

More than a hundred thousand individuals in Europe are yearly diagnosed with kidney cancer $[1,2]$. Renal cell carcinoma (RCC) is the most common type of kidney cancer and clear cell RCC (ccRCC) constitutes $75 \%$ of RCC cases [3]. Due to more extensive use of computed imaging, ultrasound, and magnetic resonance in diagnostics most patients with RCC are diagnosed incidentally before patients experience any classic symptoms (i.e. flank pain, haematuria, or a palpable abdominal mass) [4] and the frequency of metastatic disease at diagnosis has therefore decreased from 30 to $18 \%$ over a 5 -year period (2005 to 2009) [5]. Patients with non-metastatic disease at diagnosis have a 5 -year survival of $75-85 \%$, in contrast to only $10 \%$ in patients with metastatic disease. Among patients with non-metastatic disease at diagnosis, approximately $20-30 \%$ will later develop metastasis after nephrectomy, i.e. progression of the disease [5].

Non-metastatic patients with a high risk of tumor progression might benefit from an intensified follow-up to improve early diagnosis of tumor recurrence, and in the long run, be candidates for adjuvant treatment. Therefore, it is essential to identify those patients already at diagnosis by reliable biomarkers with high sensitivity and specificity.

Currently, the Mayo scoring system (Mayo) is used to predict outcome and treatment stratification in ccRCC [6]. Mayo combines T-stage, N-stage, tumor size, Fuhrman grade, and the presence of necrosis to create a score dividing patients into three groups: low, intermediate, and high-risk for tumor progression. Depending on the risk group, the frequency and the total follow-up time differs, ranging from 5 to 10 years [7].

Altered DNA methylation has been identified as a prognostic marker in several malignancies including ccRCC, and has been suggested as a potential target for therapy $[8,9]$. We have recently shown that increased promoter-associated DNA methylation is correlated to poorer outcomes in ccRCC $[10,11]$. Studies by others have identified selected $\mathrm{CpGs}$ with potential relevance for ccRCC prognosis [12-17]. Arai et al. and Tian et al. identified CPG island methylator phenotype (CIMP) panels, that predicted cancer-free survival and overall survival $[13,18]$. In 2015, Wei et al. presented a risk score based on five CpGs that predicted overall survival in three different ccRCC cohorts [14]. Joosten et al. [12] performed a systematic review summarizing prognostic DNA methylation biomarkers in ccRCC, and identified nine genes with strong evidence as prognostic biomarkers in ccRCC. However, few of these panels which are based on a limited number of CpGs, did show reproducible prognostic relevance in predicting risk for progress in non-metastatic patients [11]. Larger genome-wide panels of CpG sites might be needed for improved risk classification [12].

An interesting approach suggested by Thompson and Marsit [19] combines several molecular and clinical biomarkers to create a prognostic risk score for ccRCC patients. Even though the benefits of integrative analysis using different omics measurements and clinical data have started to become clear, few current risk classification models combine clinicopathological variables with molecular biomarkers.

The current project aimed to derive a classifier to estimate the risk of tumor progression after nephrectomy in patients with local ccRCC at diagnosis, by combining genome-wide methylation profiles and established clinicopathological variables. Three sets of biomarkers were considered in the prognostic modeling: clinicopathological variables, previously identified CpGs (PI-CpGs), and a set of methylation biomarkers obtained using a novel approach called Directed Cluster Analysis (DCA). These sets were used together with logistic regression to build classifiers predicting patients as either high risk for progress (HRP) or low risk for progress (LRP).

\section{Methods \\ ccRCC patient samples}

This study included 115 patients with ccRCC, diagnosed between 2001 and 2009 at the University Hospital in Umeå, Sweden. All patients were primarily treated with either radical or partial nephrectomy and followed up in accordance with the Swedish health care program for kidney cancer (median follow-up time 98 months ranging from 1-193 months) [7]. No patient included in this study received neoadjuvant and no non-metastatic patients received adjuvant treatment. Tissue samples were obtained after nephrectomy, snap-frozen in liquid nitrogen, and stored at $-80{ }^{\circ} \mathrm{C}$ until analysis. DNA was extracted from tissue samples as described previously [20].

The tumor disease was classified using the tumor-nodemetastasis (TNM) classifications system [21]. Eightyseven patients were non-metastatic (M0 i.e. TNM I-III) and 28 patients had metastatic disease (M1 i.e. TNM IV) at diagnosis. The nuclear grading was performed according to Fuhrman et al. [22] and the largest tumor diameter was measured at the CT scans. Blood samples were taken within a week before nephrectomy and analyzed for hemoglobin levels, thrombocyte particle count, calcium concentration, albumin levels, gamma-glutamyltransferase, alkaline phosphatase, and creatinine levels. The above variables were included in the analysis and are henceforth referred to as the clinical variables. Here, missing values were imputed using the mean value. 
All tumors were classified according to the Mayo scoring system, which calculates a risk score based on T-stage (T1a, T1b, T2, and T3-T4), tumor size (smaller/ larger than $10 \mathrm{~cm}), \mathrm{N}$-stage $(\mathrm{NX} / \mathrm{N} 0, \mathrm{~N} 1)$, Fuhrman grade (Grade 1-2, Grade 3, Grade 4) and tumor necrosis (absent/present) (Additional file 1: Table S1) [6, 7].

Clinical follow-up data were extracted in August 2017. All patients have given informed and signed consent and the study was approved by the regional ethical review board in Umeå (Dnr 2011-156-31 M, 20110523).

\section{High-dimensional DNA methylation arrays}

The DNA methylation analysis has previously been described by Evelönn et al. [11]. In short; DNA was extracted from tissue samples, from 115 tumors and 12 tumor-free (TF) tissue samples, and bisulfite converted using the EZ DNA Methylation Kit (Zymo Research, Irvine, USA). Methylation conversion was verified by MethyLight analysis [23] and samples were assessed for DNA methylation using HumanMethylation450K BeadChip arrays (Illumina, San Diego, Ca, USA). The arrays were scanned with the HiScan array reader (Illumina). The quality of each array was evaluated with the builtin controls. The technical reproducibility of methylation array analysis was monitored by replicated samples on each array, and the R2-values ranged from 0.995 to 0.997 .

Pre-processing was performed as previously described by Degerman et al. [24]. Briefly, the fluorescence intensities were extracted using the Methylation Module (1.9.0) in the Genome Studio software (V2011.1). Pre-processing and downstream analysis was done using R (v2.15.0 and v3.4.3). Data were normalized using the BMIQ method [25] to compensate for the two different bead types used in the array. Filtering of data included the exclusion of CpG probes; located at the $\mathrm{X}$ and $\mathrm{Y}$ chromosomes, that align to multiple loci in the genome [26], located less than 3 bp from a known single nucleotide polymorphism [26], without representation on the Illumina EPIC methylation arrays or had a detection p-value larger than 0.05 or a signal from less than 3 nbeads in any sample. The analysis was focused on CpGs located within the promoter region close to the transcription start site (TSS) (i.e. the regions denoted TSS1500, TSS200, 5'UTR, and exon 1). CpGs outside these regions and CpGs located in methylation quantitative trait loci (mQTLs) [27] were also excluded from the analysis. The methylation level ( $\beta$-value) of each CpG site ranged from 0 (no methylation) to 1 (complete methylation). Differently methylated CpG sites (DMCpGs) were identified by comparing the sample's $\beta$-value with the average $\beta$-value taken over the twelve TF-samples, and an absolute $\Delta \beta_{\text {tumor-TF-tissue }}$-value $\geq 0.2$ was considered as a DM-CpG.

\section{Selection of CpGs previously associated with ccRCC prognosis}

CpG sites previously associated with ccRCC, and present on HumMeth $450 \mathrm{~K}$ arrays, where identified in five original publications and one review and included in the analysis [12-17]. The systematic review by Joosten et al. 2017, identified nine genes with strong evidence as prognostic DNA methylation-based biomarkers in ccRCC [12]. Ricketts et al. [15] listed genes known to be hypermethylated in ccRCC in the publically available Tumor Cancer Genome Atlas Kidney Renal Cell Carcinoma (TCGAKIRC) project. Forty-five genes met the criterion to be hypermethylated in relation to paired tumor-free samples, and the CpG presented as most hypermethylated for each gene was included in our analysis. Van Vlodrop et al. [16] identified a four-gene promoter methylation marker panel that was associated with cancer-specific survival and validated in the TCGA-KIRC cohort. Wei et al. [14] analyzed paired tumor and tumor-free tissue samples from 46 individuals on the Infinium HumMeth450K arrays and built a model including five CpGs estimating risk for death. Arai et al. [13] created a 16 CpG site CIMP classification panel using the Infinium $27 \mathrm{~K}$ arrays that was correlated to more aggressive tumors. Fourteen out of these CpGs were represented on the Infinium HumMeth450K arrays and included in our analysis. Wang et al. [17] identified a CpG site in the DAB2IP gene, both by Illumina $450 \mathrm{~K}$ arrays and pyrosequencing that was correlated to poorer overall survival if hypermethylated.

The CpG sites identified in these publications include 87 CpGs of which 78 were unique, nine CpGs were presented in more than one publication (Additional file 2: Table S2). All CpG biomarkers included in our analysis had to fulfill the filtering steps described above except the requirement for being located within a gene promoter. After going through the filtration steps, $64 \mathrm{CpG}$ sites previously associated with ccRCC prognosis remained and were included in the analysis (Additional file 3: Table S3).

\section{Directed cluster analysis}

CpG clusters of potential prognostic relevance were identified using the novel clustering method Directed Cluster Analysis (DCA) described below. For each CpG site, the samples were grouped using 2-means clustering. Samples belonging to the group with the highest mean $\beta$-value were labeled 1 while the other samples were labeled 0 . Hence, for each CpG site, a binary profile vector was constructed. CpGs with less than $10 \%$ of the samples in the smallest group were removed from further analysis. The difference between the mean $\beta$-values in the two groups was calculated for each $\mathrm{CpG}$ and sites with an absolute difference lower than 0.2 were excluded from 
downstream analysis. The remaining CpGs were clustered using $\mathrm{k}$-means with $\mathrm{k}=40$. Note that CpGs with similar binary partition of the samples are more likely to end up in the same cluster. For each cluster and sample, a robust consensus variable was obtained by calculating the mean $\beta$-value of all sites included in the cluster. The consensus variables were treated as biomarkers. The DCA method workflow is summarized in Fig. 1a, b.

\section{Classification}

The classification was used to estimate the risk of metastasis within 5 years among patients with non-metastatic disease at diagnosis. The inclusion criterion was at least a 5-year follow-up, which was met by 78 non-metastatic patients. Fifty-eight patients remained non-metastatic (M0-PF), while 20 patients progressed (M0-P) during the 5-year follow-up. Three sets of biomarkers were considered: the clinicopathological variables (clinical), the previously identified ccRCC associated methylation sites (PI-CpGs), and the consensus variables identified by directed cluster analysis (DCA). The sets were used individually and combined in six sets: clinical, PI-CpGs, DCA, clinical+PI-CpGs, clinical+DCA, and clini$\mathrm{cal}+\mathrm{PI}-\mathrm{CpGs}+\mathrm{DCA}$. For each set, a classifier was built on the first five Principal Components (PC) using logistic regression. The PCs' were obtained using standardized variables, except for the categorical and ordinal variables that were mean-centered. The classifiers were evaluated by the specificity observed at a fixed $85 \%$ sensitivity.

\section{Statistical analysis}

Differences between patient groups were analyzed using the Mann-Whitney $U$ test for continuous variables and a

Filtration of $\mathrm{CpG}$ sites as described in methods section



Cluster each CpG individually into two groups such that group 1 always have higher mean value.

Exclude sites with skew distribution and with absolute difference in methylation lower than 0.2 .



Construct the consensus variables by calculating the mean $\beta$ for each cluster

\section{Build Classifier}

b

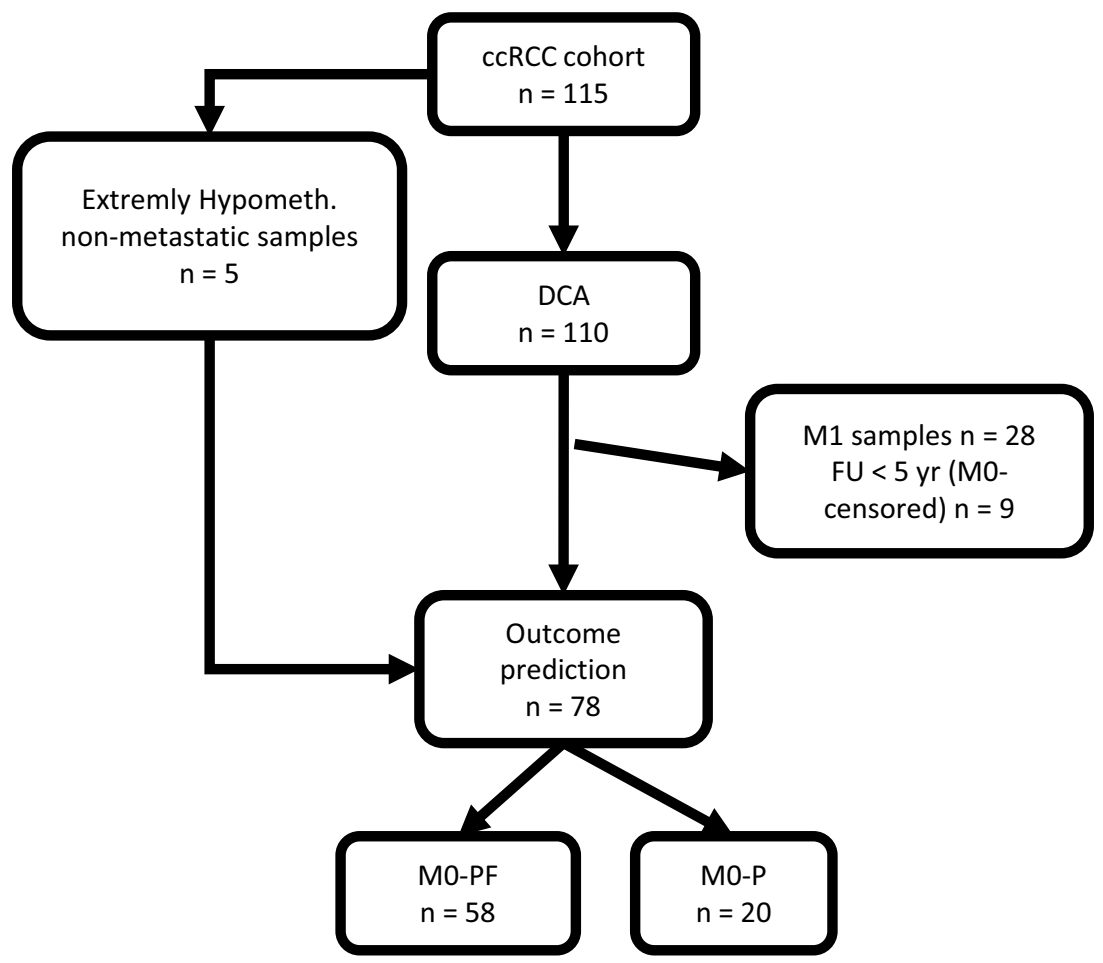

Fig. 1 Analysis workflow. a DCA consensus cluster workflow. Showing the steps for the creation of consensus variables using Directed Cluster Analysis (DCA). b Patient inclusion in analysis steps 
the Chi-square test for categorical variables. The calculations were performed in R v3.4.3. Agreement between the Mayo $\left(\mathrm{MAYO}_{\text {high/intermediate }}\right.$ and $\mathrm{MAYO}_{\text {low }}$ risk) and the triple classifier (high risk and low risk) was measured by the Cohen's kappa score in R-package psych.

Receiver operating characteristic (ROC) analysis and the area under the curve (AUC) calculations were done using R-package pROC, Mayo scores, and the posterior probabilities of the six classifiers. The ROC curves were compared using the methodology described by DeLong et al. [28].

The cumulative incidence of progress within 5 years from diagnosis $\left(\mathrm{CIP}_{5 \mathrm{yr}}\right)$ was estimated by Kaplan-Meier survival tables. Differences in survival distributions between subgroups of samples were calculated using the log-rank test. Survival analysis was performed using the Statistical Package for the Social Sciences (SPSS Inc., Chicago, IL) software version 24.

\section{Results}

\section{Descriptive analysis of the ccRCC cohort}

A total of 115 patients were included in this study, 57\% were men, and the median age was $65( \pm 11.6)$ years at diagnosis. The patients were divided into three groups; non-metastatic progression free $(\mathrm{M} 0-\mathrm{PF}, \mathrm{n}=58)$; nonmetastatic with progression within 5 years (M0-P; $\mathrm{n}=20)$, and metastasized at diagnosis $(M 1 ; n=28)$. Nine non-metastatic patients (M0-censored) were excluded from the risk classification models as 5 years follow-up was not reached e.g. due to the death of other diseases

Table 1 Clinicopathological variables and their relation to ccRCC progression

\begin{tabular}{|c|c|c|c|c|c|c|}
\hline Variable & $M 0-P F n=58$ & $M 0-P n=20$ & $M 1 n=28$ & $\begin{array}{l}\text { Mo-PF vs M0-P } \\
\text { p-value }\end{array}$ & $\begin{array}{l}\text { M0-PF vs M1 } \\
\text { p-value }\end{array}$ & $\begin{array}{l}\text { Mo-P } \\
\text { vs M1 } \\
\text { p-value }\end{array}$ \\
\hline \multicolumn{7}{|l|}{ Gender } \\
\hline Male & 32 & 10 & 21 & 0.888 & 0.125 & 0.139 \\
\hline Female & 26 & 10 & 7 & & & \\
\hline \multicolumn{7}{|l|}{ Morphological grade $^{a}$} \\
\hline G1 & 12 & 1 & 0 & 0.002 & $<0.001$ & 0.394 \\
\hline G2 & 30 & 6 & 7 & & & \\
\hline G3 & 15 & 8 & 8 & & & \\
\hline G4 & 1 & 5 & 12 & & & \\
\hline \multicolumn{7}{|l|}{ TNM } \\
\hline 1 & 39 & 5 & 0 & $<0.001$ & $<0.001$ & $<0.001$ \\
\hline$\|$ & 10 & 2 & 0 & & & \\
\hline III & 9 & 13 & 0 & & & \\
\hline IV & 0 & 0 & 28 & & & \\
\hline \multicolumn{7}{|l|}{ Mayo } \\
\hline Low & 29 & 3 & & 0.006 & - & - \\
\hline Intermediate/high & 29 & 17 & & & & \\
\hline Age (years) & $65.6(11.6)$ & $64.2(11.9)$ & $63.0(11.0)$ & 0.590 & 0.345 & 0.706 \\
\hline Albumin (g/L) & $40.6(4.1)$ & $38.6(7.2)$ & $36.7(4.5)$ & 0.299 & $<0.001$ & 0.121 \\
\hline Alkaline phosphatase $(\mu k a t / L)^{b}$ & $1.9(2.0)$ & $2.2(1.5)$ & $5.1(6.6)$ & 0.745 & $<0.001$ & 0.026 \\
\hline Calcium $(\mathrm{mmol} / \mathrm{L})^{c}$ & $2.35(0.14)$ & $2.32(0.13)$ & $2.46(0.26)$ & 0.358 & 0.212 & 0.129 \\
\hline Creatinine $(\mu \mathrm{mol} / \mathrm{L})$ & $79.6(16.3)$ & $86.0(32.4)$ & $93.3(27.6)$ & 0.837 & 0.015 & 0.300 \\
\hline Gamma glutamyltanseferase $(\mu k a t / L)^{d}$ & $0.76(1.17)$ & $0.83(1.21)$ & $2.23(3.06)$ & 0.401 & $<0.001$ & 0.002 \\
\hline Hemoglobin (g/L) & $137.6(17.3)$ & $121.1(22.0)$ & $116.9(19.1)$ & 0.002 & $<0.001$ & 0.331 \\
\hline Thromobocyte particle count $\left(10^{9} / \mathrm{L}\right)$ & $251.7(77.7)$ & $316.5(175.4)$ & $365.3(136.8)$ & 0.112 & $<0.001$ & 0.090 \\
\hline Tumor diameter (mm) & $56.5(31.2)$ & $95.0(44.8)$ & $108.3(35.7)$ & $<0.001$ & $<0.001$ & 0.262 \\
\hline
\end{tabular}

Mean values and standard deviation (SD) are reported for each continuous variable. Chi-square tests were used for testing independence between categorical variables and Mann-Whitney $\mathrm{U}$ tests were used for comparisons between continuous variables. M0 and M1 denote non-metastatic and metastatic patients at diagnosis respectively, while PF and P denote progress free patients and patients with progress within 5 years respectively

a One missing value in group M1

b Three missing values. One value is missing in each of MO-PF, M0-P and M1

c One missing value in M0-PF

d Four missing values. Three in M0-PF and one in M1 
(Fig. 1b). No significant differences in age or gender were observed between the subgroups (Table 1).

Individual analysis of clinical and methylation variables. Twelve clinical variables were compared in the groups M0-PF and M0-P. The tumor diameter was significantly larger in the M0-P group than in the M0-PF group at diagnosis $(\mathrm{p}<0.001), 95.0 \mathrm{~mm}$ and $56.5 \mathrm{~mm}$, respectively. Moreover, the distributions of morphological grade $(\mathrm{p}=0.002)$ and TNM stage $(\mathrm{p}<0.001)$ differed in M0-PF and M0-P patients, with a higher grade/TNM stage in the M0-P patients. The hemoglobin value was significantly higher $(\mathrm{p}=0.002)$ in M0-PF (mean $137.6 \mathrm{~g} / \mathrm{l})$ than in M0-P (mean $121.1 \mathrm{~g} / \mathrm{l}$ ) patients at diagnosis. For the remaining clinical variables, no significant differences were observed (Table 1 ).

Next, differences in methylation between M0-P and M0-PF were investigated. For the 64 previously identified sites (PI-CpGs), 15 (23.4\%) sites were significantly more methylated in the M0-P group compared to the M0-PF group (Additional file 3: Table S3).

Before the DCA analysis, an overview of all the samples' methylation profiles was performed. Five non-metastatic samples showed an extreme number of hypomethylated $(\Delta \beta \leq-0,2) \quad \mathrm{CpG}$ and were excluded from the DCA clustering process to avoid heavily unbalanced distributions in the first step in the DCA procedure. However, these samples were included in the downstream analysis after the consensus variables were constructed (Fig. 1b).

Applying the DCA method yielded 40 DNA methylation cluster variables (Additional file 4: Table S4). Six (15\%) of these variables showed a significantly higher methylation level in the M0-P group compared to the M0-PF group (Additional file 5: Table S5).

\section{Classification of non-metastatic ccRCC patients}

The objective was to build classification models that can be used to predict tumor progression among non-metastatic ccRCC patients at diagnosis, i.e. either M0-PF or M0-P. Six classification models were constructed and evaluated against the Mayo classification. Mayo classified 32 patients as low-risk, 36 patients as intermediate-risk, and 10 patients as high-risk. To calculate the specificity and sensitivity of Mayo, we pooled the Mayo intermediate and Mayo high groups motivated by identical clinical follow-up scheme for these patients [7]. The pooled group was denoted Mayo intermediate/high. Mayo showed $85 \%$ sensitivity and $50 \%$ specificity in our cohort.

The six classifiers were derived using logistic regression and the first five principal components taken from one or several of the considered data sets i.e. clinical, PI-CpGs, DCA, clinical+PI-CpGs, clinical+DCA, and clinical + PI-CpGs + DCA. Each classifier predicted the patients as either low risk for progress (LRP) or high risk for progress (HRP) (Additional file 6: Table S6). To enable comparison with Mayo, the classifiers were evaluated by considering the specificity when the sensitivity was fixed at $85 \%$ by controlling the cutoff of the predicted posterior probabilities. The expected specificity observed by chance for a non-informative random data was tested through simulation and was estimated to $11 \%$.

The performances of the classifiers using the clinical, PI-CpGs, and DCA data alone were compared with Mayo. The clinical, PI-CpGs, and DCA classifiers had $43 \%, 59 \%$ and $43 \%$ specificity respectively (Table 2 ). The deviated predictions suggest that the data sets contain complementary information and that better predictive models can be obtained by combining several data sets.

Accordingly, the triple classifier using data from all three data sets, i.e. 12 clinical variables, 64 PI-CpGs, and 40 DCA variables had the highest specificity (64\%) (Table 2). This triple classifier correctly identified 37 of 58 patients without progression and 17 of 20 patients with tumor progression. Classification of new ccRCC patients using the triple classifier can be performed using the $\mathrm{R}$ script found at: https://github.com/LindaVi/ccRCC-class ification. To impute missing values, mean values based on our cohort for all variables included in the model, are supplied along with the script.

Table 2 Performance of the considered classifiers and the Mayo Scoring system

\begin{tabular}{llll}
\hline & Classifier & Sensitivity (\%) & Specificity (\%) \\
\hline A & Mayo scoring system (Mayo) & 85 & 50 \\
B & Clinicopathological variables (Clinical) & 85 & 43 \\
C & Identified prognostic biomarker CpGs (PI-CpGs) & 59 \\
D & Consensus variables (DCA) & 85 & 43 \\
E & Clinical +PI-CpGs & 85 & 55 \\
F & Clinical +DCA & 85 & 53 \\
G & Clinical +PI-CpGs + DCA (the triple classifier) & 85 & 64 \\
\hline
\end{tabular}


ROC-curves were made for all classifiers and no significant differences ( $p>0.05$ for all pairwise comparisons) were shown between the overall AUC-values (Additional file 8: Figure S1).

\section{Similarities between models}

A sensitivity of $85 \%$ entails that each of the models correctly classified 17 of the 20 patients with tumor progression within 5 years. Sixty-five percent of the M0-P patients and $21 \%$ of the M0-PF patients were correctly classified by all seven models, including Mayo. Moreover, $47 \%$ of the patients (independent of true outcome) were classified the same by all classifiers (Additional file 6: Table S6).

As expected, methods relying on only clinical data (clinical and Mayo) gave similar output (Fig. 2a and Additional file 6: Table S6), where $87 \%$ of the patients were classified identically by the methods. The DCA and PI-CpGs classifiers that rely on only methylation data, showed identical classification output in $81 \%$ of the patients (Fig. 2a, b). The similarities in the classification of the triple classifier compared to every single subset; clinical, PI-CpGs and DCA, showed an overlap in $72 \%$, $83 \%$, and $74 \%$ respectively (Fig. 2 b).

The triple classifier showed a moderate agreement with the Mayo classification, with Cohen's kappa $=0.49$ and with $74 \%$ of the samples classified identically (Fig. 2c, d and Additional file 7: Table S7).

\section{Survival analysis}

Cumulative incidence of progress (CIP) analysis was used to investigate the prognostic relevance for the triple classifier and Mayo in 78 non-metastatic ccRCC patients. Both classifiers divided the patients into two groups: low risk for progress and high risk for progress. The triple classifier was better than Mayo to prognosticate

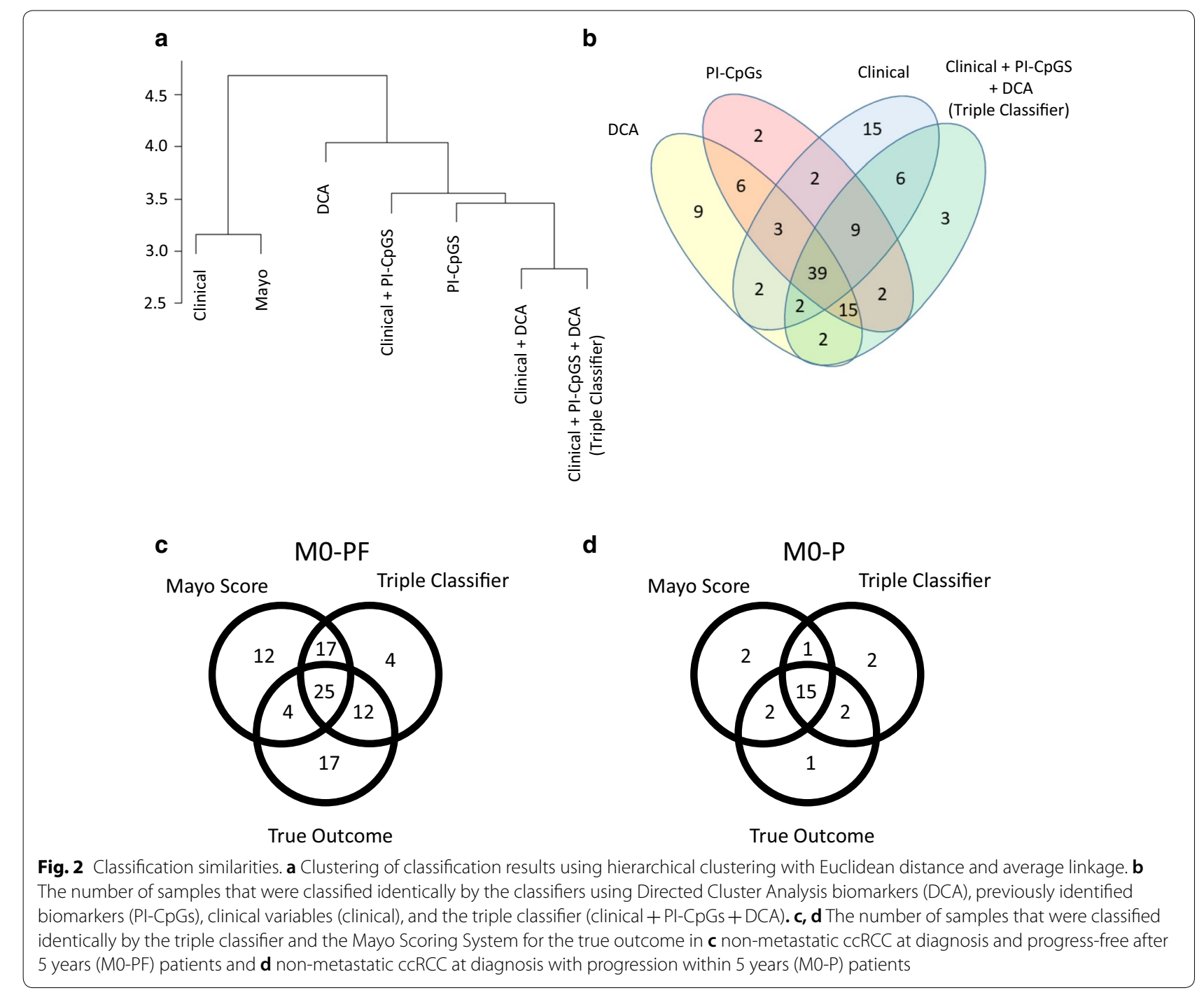




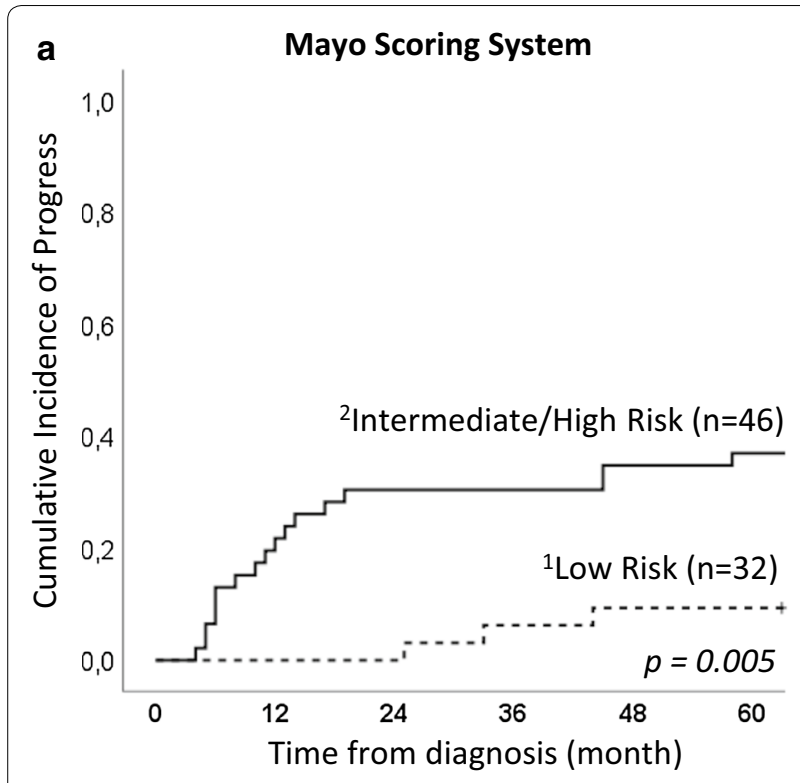

No. at risk (cum. events)

$\begin{array}{llllll}{ }^{132}(0) & 32(0) & 32(0) & 30(2) & 29(3) & 29(3) \\ { }^{2} 46(0) & 36(10) & 32(14) & 32(14) & 30(16) & 29(17)\end{array}$

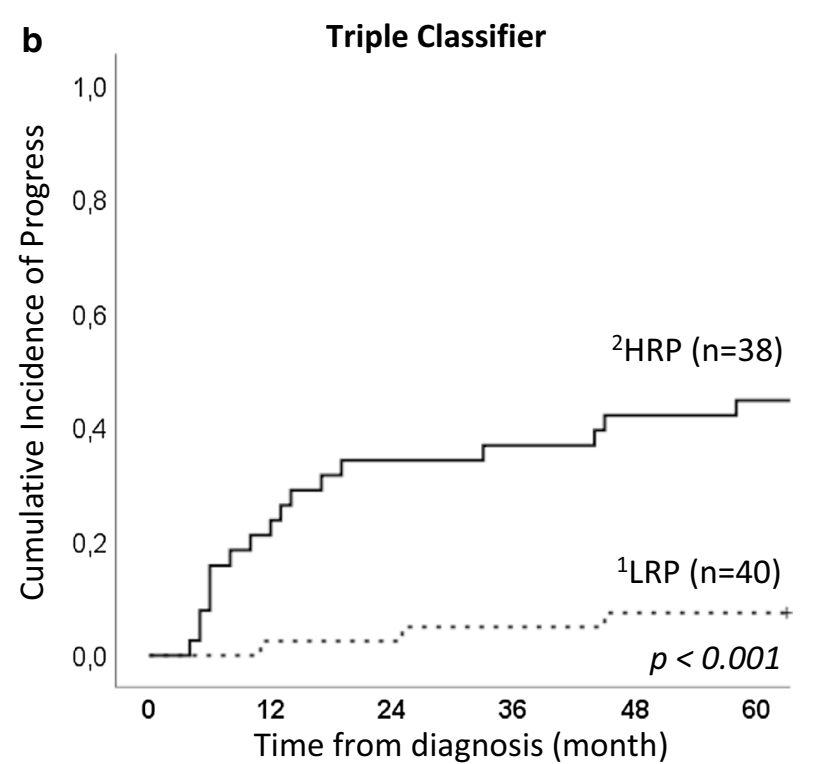

No. at risk (cum. events)

$\begin{array}{llllll}{ }^{140(0)} & 39(1) & 38(2) & 38(2) & 37(3) & 37(3) \\ 238(0) & 29(9) & 25(13) & 24(14) & 22(16) & 21(17)\end{array}$

Fig. 3 Cumulative incidence of progress $\left(\mathrm{pCIP}_{5 \mathrm{y}}\right)$. Seventy-eight non-metastatic tumors were classified using a the Mayo Scoring System (Mayo) and $\mathbf{b}$ the triple classifier (clinical + PI-CpGs + DCA) at diagnosis. The $\mathrm{pCIP}_{5 y}$ were compared in the risk groups. Log-rank $p$-values are presented

progress $\left(\mathrm{pCIP}_{5 \mathrm{yr}} 9.4 \%\right.$ low risk Mayo vs $7.5 \% \mathrm{LRP}_{\text {triple clas- }}$ sifier $)\left(\mathrm{pCIP}_{5 \mathrm{yr}} 37 \%\right.$ intermediate/high risk $_{\text {Mayo }}$ vs $44.7 \%$ $\mathrm{HRP}_{\text {triple classifier }}$ (Fig. 3).

\section{Discussion}

DNA methylation alterations are early events in tumor progression and has appeared as a molecular biomarker candidate for improved risk classification [11-17]. Our study shows that the prediction of tumor progression within 5 years after nephrectomy in non-metastatic ccRCC patients at diagnosis was improved by using bioinformatics modeling to combine clinicopathological variables with methylation signatures.

One-third of non-metastatic ccRCC patients at diagnosis are at risk for later tumor progress [5]. It is of importance to identify patients with a high-risk for tumor progression, since they may benefit from a more intensive follow-up and adjuvant therapy. As reviewed by Bai et al. [29] several adjuvant studies using tyrosine kinase inhibitors (TKI) have been performed in patients with locally advanced non-metastatic RCC. The conclusion drawn from this systematic review is that there might be ccRCC patients that benefit from adjuvant treatment, as described in the S-TRAC study showing longer disease-free time but not for overall survival, while other TKI studies showed no survival benefit [29, 30] Deepened knowledge of ccRCC biology is important to identify new therapeutic strategies for high-risk patients. New ongoing phase 3 adjuvant immunotherapy trials might theoretically be more successful since these therapies unblock the hampering of the patient's immune cells by the tumors [31].

Risk classification strategies which include both clinical and molecular biomarkers have the potential to improve sensitivity and specificity and more effectively identify the patients at high risk for progress which may benefit from modern adjuvant therapies, despite the common side-effects.

Our population-based ccRCC cohort represents wellcharacterized patients with long follow-up time. We performed genome-wide methylation analysis on the diagnostic tumor samples and included both M0 and M1 samples in the DCA modelling.

Among the 78 non-metastatic ccRCC at diagnosis, the currently used Mayo showed a sensitivity of $85 \%$ and a specificity of $50 \%$ in predicting tumor progression. We tested six different classifiers including clinical and/or methylation derived variables at a fixed desirably high sensitivity of $85 \%$ to allow comparison with Mayo, and also motivated by the clinical need for high sensitivity in risk classification. The specificity at the fixed sensitivity of $85 \%$ was considerably higher for the 
triple classifier than for Mayo (64\% vs. $50 \%)$, although the overall ROC curve analysis could not significantly discriminate between the classifiers' AUC. Moreover, the triple classifier showed a more accurate prediction of progress than Mayo in the cumulative incidence of progress analysis.

Altogether, the addition of methylation biomarkers to the currently used clinical variables improved the prediction of progress among non-metastatic patients at diagnosis. Thompson and Marsit [19] also combined clinical variables and molecular biomarkers, including both methylation and gene-expression profiles. Their study supports our conclusion that methylation analysis has the potential to improve risk classification in ccRCC.

Biomarkers derived from DNA methylation analysis have several advantages, e.g. DNA methylation alterations are stable, do not require adapted sample handling, and DNA is routinely extracted in the clinic. DNA methylation classifiers are in clinical use as a prognostic marker in other malignancies. Jaunmuktane et al. used the classifier presented by Capper et al. in combination with established methods (i.e. histological assessment and conventional molecular testing) to diagnose CNS tumors. Methylation-based classification contributed to a more accurate and clinically relevant diagnosis, most pronounced in unusual, non-specific, or non-representative histological cases [32, 33]. Bioinformatics pipelines for methylation array data analysis in CNS tumors are being introduced at clinical diagnostic laboratories [32, 34]. Although methylation analyses have shown prognostic value in ccRCC [12] they are still not used in clinical diagnosis.

An alternative classifier using gene expression data, ClearCode34, is of prognostic relevance in several ccRCC cohorts as reviewed by Ghatalia and Rathmell [35]. Expression levels of 34 genes were analyzed and tumors were classified as either low-risk ccA or high-risk ccB. Notably, the same tumor could be categorized as both $\mathrm{ccA}$ and $\mathrm{ccB}$ due to tumor heterogeneity in expression levels. We have previously shown that DNA methylation patterns in ccRCCs are heterogeneous between different patients, but within individual tumors, the methylation pattern is homogenous [11]. This was further supported by the study of Wei et al. who classified multiple samples within each tumor and showed that $90 \%$ of tumors were correctly classified by DNA methylation-based classification [14], suggesting DNA methylation as an attractive and stable biomarker.

The proposed DCA-method is a novel unsupervised approach for identifying potential biomarker panels. Hence, the method does not use class information, in our case information on progress, to derive the biomarkers. An advantage with an unsupervised approach is that it can capture biological signatures also when the classes of interest are heterogeneous.

The DCA variables are obtained by taking the average beta-value over a large number of $\mathrm{CpGs}$, which makes them less sensitive to noise compared to single $\mathrm{CpG}$ biomarkers. Future studies on independent ccRCC cohorts are of importance for confirming the prognostic relevance of our defined risk classification panel. We encourage researchers to independently evaluate the proposed triple classifier, utilizing standard clinical data and methylation data from either the Infinium Human Methylation $450 \mathrm{k}$ BeadChip or Infinium MethylationEPIC arrays.

We believe that our study shows the potential in combining clinicopathological variables with methylation signatures to improve current risk classification of nonmetastatic ccRCC.

\section{Conclusions}

A combination of clinicopathological and epigenetic variables, i.e. DNA methylation, have benefits in predicting survival of patients with ccRCC, compared to the currently used Mayo classifier. The triple classifier has the potential to enhance specificity in identifying ccRCC patients with a high risk of tumor progression and improve treatment stratification.

\section{Supplementary information}

Supplementary information accompanies this paper at https://doi. org/10.1186/s12967-020-02608-1.

Additional file 1: Table S1. The Mayo scoring algorithm (6), used in the clinic to estimate the risk of tumor progression.

Additional file 2: Table S2. CpGs with potential relevance for ccRCC prognosis identified in the literature (PI-CpGs).

Additional file 3: Table S3. CpGs with potential relevance for ccRCC prognosis identified in the literature (PI-CpGs). Mean beta values are presented for M0-PF and M0-P patients in our cohort, as well as p-values derived using the Mann-Whitney $U$ test.

Additional file 4: Table S4.CpGs included in the 40 consensus variables identified by DCA.

Additional file 5: Table S5. Mean beta values for the 40 consensus variables identified by DCA for M0-P and M0-PF patients together with $\mathrm{p}$-value comparing average methylation for each cluster using the MannWhitney $U$ test.

Additional file 6: Table S6. Prediction results for the considered classifiers and the Mayo scoring system for the 78 non-metastatic patients at diagnosis. Each classifier predicted the patients as either low risk for progress $(\mathrm{LRP}=0)$ or high risk for progress $(\mathrm{HRP}=1)$.

Additional file 7: Table S7. Classification results for the Mayo scoring classifier (Low Risk or Intermediate/High Risk) and the triple classifier (LRP or HRP) in relation to the true outcome (MO-PF or M0-P).

Additional file 8: Figure S1. Receiver operating characteristic (ROC) analysis and the area under the curve (AUC) analysis based on Mayo scores, and the posterior probabilities of the six classifiers. 


\begin{abstract}
Abbreviations
RCC: Renal cell carcinoma; ccRCC: Clear cell renal cell carcinoma; CpG: Cytosine-phosphate-Guanine; CIMP: CpG island methylator phenotype; PICpG: Previously identified CpG; DCA: Directed cluster analysis; HRP: High risk for progress; LRP: Low risk for progress; TNM: Tumor-node metastasis; M0: Nonmetastatic (TNM I-III); M1: Metastatic; TSS: Transcription start site; DM-CpG: Differently methylated CpG; TCGA-KIRC:Tumor Cancer Genome Atlas Kidney Renal Cell Carcinoma; mQTLs: Methylation quantitative trait loci; MO-PF: Non metastatic without tumor progression within five year; M0-P: Non-metastatic tumor with progress within five year; PC: Principal components; CIP: Cumulative incidence of progress.
\end{abstract}

\section{Authors' contributions}

PR and SD conceived the original idea and all authors contributed to the design of the study. EAE performed the experiments and $M$. L conducted the preprocessing of the data. LV, DK, PR proposed the DCA method. LV, DK, ML, $\mathrm{XL}, \mathrm{SD}$ and $\mathrm{EAE}$ analyzed the data. All authors discussed the results. EAE, LV, $\mathrm{SD}$, and PR wrote the first draft of the manuscript with contribution from all co-authors. All authors read and approved the final manuscript.

\section{Funding}

Open Access funding provided by Umeå University. Supported by grants from the Faculty of Science and Technology, the Medical Faculty of Umeå University, Lion's Cancer Research Foundation, Umeå, the Kempe research foundation, the Swedish Research Council, and Uppsala-Umeå Comprehensive Cancer Consortium. Financial support was provided through a regional agreement between Umeå University and Västerbotten County Council on cooperation in the field of Medicine, Odontology, and Health.

\section{Availability of data and materials}

The methylation datasets analyzed during the current study are available in the NCBI Gene Expression Omnibus (GEO) repository [GSE113501]

\section{Ethics approval and consent to participate}

All patients have given informed consent, and the study was approved by the regional Ethical review board in Umeå (Dnr 2011-156-31 M, 20110523), Sweden.

\section{Consent for publication}

Not applicable.

\section{Competing interests}

The authors declare that they have no competing interests.

\section{Author details}

${ }^{1}$ Department of Medical Biosciences, Pathology, Umeå University, 901 87 Umeå, Sweden. ${ }^{2}$ Department of Mathematics and Mathematical Statistics, Umeå University, 90187 Umeå, Sweden. ${ }^{3}$ Department of Statistics, USBE, Umeå University, Umeå, Sweden. ${ }^{4}$ Department of Surgical and Perioperative Sciences, Urology and Andrology, Umeå University, Umeå, Sweden. ${ }^{5}$ Department of Clinical Microbiology, Umeå University, Umeå, Sweden.

Received: 17 May 2020 Accepted: 5 November 2020

Published online: 13 November 2020

\section{References}

1. Ferlay J, Colombet M, Soerjomataram I, Dyba T, Randi G, Bettio M, Gavin A, Visser O, Bray F. Cancer incidence and mortality patterns in Europe: estimates for 40 countries and 25 major cancers in 2018. Eur J Cancer. 2018;103:356-87.

2. Capitanio U, Bensalah K, Bex A, Boorjian SA, Bray F, Coleman J, Gore JL, Sun M, Wood C, Russo P. Epidemiology of renal cell carcinoma. Eur Urol. 2019;75:74-84.

3. Kovacs G, Akhtar M, Beckwith BJ, Bugert P, Cooper CS, Delahunt B, Eble $J N$, Fleming S, Ljungberg B, Medeiros LJ, et al. The Heidelberg classification of renal cell tumours. J Pathol. 1997;183:131-3.

4. Thorstenson A, Bergman M, Scherman-Plogell AH, Hosseinnia S, Ljungberg B, Adolfsson J, Lundstam S. Tumour characteristics and surgical treatment of renal cell carcinoma in Sweden 2005-2010: a populationbased study from the National Swedish Kidney Cancer Register. Scand J Urol. 2014:48:231-8.

5. Dabestani S, Thorstenson A, Lindblad P, Harmenberg U, Ljungberg $B$, Lundstam S. Renal cell carcinoma recurrences and metastases in primary non-metastatic patients: a population-based study. World J Urol. 2016:34:1081-6.

6. Leibovich BC, Blute ML, Cheville JC, Lohse CM, Frank I, Kwon ED, Weaver AL, Parker AS, Zincke H. Prediction of progression after radical nephrectomy for patients with clear cell renal cell carcinoma: a stratification tool for prospective clinical trials. Cancer. 2003;97:1663-71.

7. Regionalt Cancercentrum. Nationellt vårdprogram njurcancer. 2019.

8. Esteller M. Epigenetics in cancer. N Engl J Med. 2008;358:1148-59.

9. Morris MR, Maher ER. Epigenetics of renal cell carcinoma: the path towards new diagnostics and therapeutics. Genome Med. 2010;2:59.

10. Evelonn EA, Degerman S, Kohn L, Landfors M, Ljungberg B, Roos G. DNA methylation status defines clinicopathological parameters including survival for patients with clear cell renal cell carcinoma (ccRCC). Tumour Biol. 2016;37:10219-28.

11. Evelönn EA, Landfors $M$, Haider Z, Köhn L, Ljungberg B, Roos G, Degerman S. DNA methylation associates with survival in non-metastatic clear cell renal cell carcinoma. BMC Cancer. 2019;19:65.

12. Joosten SC, Deckers IA, Aarts MJ, Hoeben A, van Roermund JG, Smits KM, Melotte V, van Engeland M, Tjan-Heijnen VC. Prognostic DNA methylation markers for renal cell carcinoma: a systematic review. Epigenomics. 2017;9:1243-57.

13. Arai E, Chiku S, Mori T, Gotoh M, Nakagawa T, Fujimoto H, Kanai Y. Single-CpG-resolution methylome analysis identifies clinicopathologically aggressive CpG island methylator phenotype clear cell renal cell carcinomas. Carcinogenesis. 2012;33:1487-93.

14. Wei JH, Haddad A, Wu KJ, Zhao HW, Kapur P, Zhang ZL, Zhao LY, Chen ZH, Zhou YY, Zhou JC, et al. A CpG-methylation-based assay to predict survival in clear cell renal cell carcinoma. Nat Commun. 2015;6:8699.

15. Ricketts CJ, Hill VK, Linehan WM. Tumor-specific hypermethylation of epigenetic biomarkers, including SFRP1, predicts for poorer survival in patients from the TCGA Kidney Renal Clear Cell Carcinoma (KIRC) project. PLOS ONE. 2014;9:e85621.

16. van Vlodrop IJ, Joosten SC, de Meyer T, Smits KM, Van Neste L, Melotte $V$, Baldewijns $M$, Schouten $L$, van den Brandt PA, Jeschke J, et al. A fourgene promoter methylation marker panel consisting of GREM1, NEURL, LAD1 and NEFH predicts survival of clear cell renal cell cancer patients. Clin Cancer Res. 2016:23:2006-18.

17. Wang ZR, Wei JH, Zhou JC, Haddad A, Zhao LY, Kapur P, Wu KJ, Wang $B$, Yu YH, Liao B, et al. Validation of DAB2IP methylation and its relative significance in predicting outcome in renal cell carcinoma. Oncotarget. 2016:7:31508-19.

18. Tian Y, Arai E, Gotoh M, Komiyama M, Fujimoto H, Kanai Y. Prognostication of patients with clear cell renal cell carcinomas based on quantification of DNA methylation levels of CpG island methylator phenotype marker genes. BMC Cancer. 2014;14:772.

19. Thompson JA, Marsit CJ. A methylation-to-expression feature model for generating accurate prognostic risk scores and identifying disease targets in clear cell kidney cancer. Pac Symp Biocomput. 2017;22:509-20.

20. Svenson $U$, Ljungberg B, Roos G. Telomere length in peripheral blood predicts survival in clear cell renal cell carcinoma. Cancer Res. 2009;69:2896-901.

21. Sobin LHGM, Wittekind C. TNM classification of malignant tumors. UICC International Union Against Cancer.: Wiley-Blackwell; 2009.

22. Fuhrman SA, Lasky LC, Limas C. Prognostic significance of morphologic parameters in renal cell carcinoma. Am J Surg Pathol. 1982;6:655-63.

23. Weisenberger DJ, Campan M, Long TI, Kim M, Woods C, Fiala E, Ehrlich M, Laird PW. Analysis of repetitive element DNA methylation by MethyLight. Nucleic Acids Res. 2005;33:6823-36.

24. Degerman S, Landfors M, Siwicki JK, Revie J, Borssen M, Evelonn E, Forestier E, Chrzanowska KH, Ryden P, Keith WN, Roos G. Immortalization of T-cells is accompanied by gradual changes in CpG methylation resulting in a profile resembling a subset of T-cell leukemias. Neoplasia. 2014;16:606-15.

25. Teschendorff AE, Marabita F, Lechner M, Bartlett T, Tegner J, GomezCabrero D, Beck S. A beta-mixture quantile normalization method for 
correcting probe design bias in Illumina Infinium 450 k DNA methylation data. Bioinformatics. 2013;29:189-96.

26. Nordlund J, Backlin CL, Wahlberg P, Busche S, Berglund EC, Eloranta ML, Flaegstad T, Forestier E, Frost BM, Harila-Saari A, et al. Genome-wide signatures of differential DNA methylation in pediatric acute lymphoblastic leukemia. Genome Biol. 2013;14:r105.

27. Gaunt TR, Shihab HA, Hemani G, Min JL, Woodward G, Lyttleton O, Zheng J, Duggirala A, McArdle WL, Ho K, et al. Systematic identification of genetic influences on methylation across the human life course. Genome Biol. 2016;17:61.

28. DeLong ER, DeLong DM, Clarke-Pearson DL. Comparing the areas under two or more correlated receiver operating characteristic curves: a nonparametric approach. Biometrics. 1988;44:837-45.

29. Bai Y, Li S, Jia Z, Ding Y, Gu C, Yang J. Adjuvant therapy for locally advanced renal cell carcinoma: a meta-analysis and systematic review. Urol Oncol. 2018;36:79.e71-79.e10.

30. Ravaud A, Motzer RJ, Pandha HS, George DJ, Pantuck AJ, Patel A, Chang YH, Escudier B, Donskov F, Magheli A, et al. Adjuvant Sunitinib in high-risk renal-cell carcinoma after nephrectomy. N Engl J Med. 2016;375:2246-54.

31. Singla N, Freifeld $Y$, Ghandour RA, Hammers HJ. Rational approaches to treatment duration with immunotherapy in metastatic renal cell carcinoma. Eur Urol Focus. 2020;6:31-3.
32. Jaunmuktane Z, Capper D, Jones DTW, Schrimpf D, Sill M, Dutt M, Suraweera N, Pfister SM, von Deimling A, Brandner S. Methylation array profiling of adult brain tumours: diagnostic outcomes in a large, single centre. Acta Neuropathol Commun. 2019;7:24.

33. Capper D, Jones DTW, Sill M, Hovestadt V, Schrimpf D, Sturm D, Koelsche C, Sahm F, Chavez L, Reuss DE, et al. DNA methylation-based classification of central nervous system tumours. Nature. 2018;555:469.

34. Capper D, Stichel D, Sahm F, Jones DTW, Schrimpf D, Sill M, Schmid S, Hovestadt V, Reuss DE, Koelsche C, et al. Practical implementation of DNA methylation and copy-number-based CNS tumor diagnostics: the Heidelberg experience. Acta Neuropathol. 2018;136:181-210.

35. Ghatalia P, Rathmell WK. Systematic review: clearcode 34-a validated prognostic signature in clear cell renal cell carcinoma (ccRCC). Kidney Cancer. 2018;2:23-9.

\section{Publisher's Note}

Springer Nature remains neutral with regard to jurisdictional claims in published maps and institutional affiliations.
Ready to submit your research? Choose BMC and benefit from:

- fast, convenient online submission

- thorough peer review by experienced researchers in your field

- rapid publication on acceptance

- support for research data, including large and complex data types

- gold Open Access which fosters wider collaboration and increased citations

- maximum visibility for your research: over $100 \mathrm{M}$ website views per year

At BMC, research is always in progress.

Learn more biomedcentral.com/submissions 